\title{
Traffic Routing Oligopoly
}

\author{
Dávid Csercsik • Balázs Sziklai
}

Received: date / Accepted: date

\begin{abstract}
The purpose of this paper is to introduce a novel family of transferable utility games related to congested networks. We assume that players are traffic coordinators, who explicitly route their deliveries in the network. The costs of the players are determined by the total latency of the deliveries, which in turn can be calculated by the edge latency functions. Since the edge latency functions assign a latency value to the total flow on the corresponding edge, as cooperating players redesign their routing in order to minimize their overall cost, outsiders will be affected as well. This gives rise to externalities therefore the resulting game is described in partition function form. We show that cooperation may imply both negative and positive externalities in the defined game. We assume that coalitions may determine their routing according to different predictive strategies. We show that the increasing order of predictive strategies may converge to a Nash equilibrium, although convergence is not guaranteed, even if a unique Nash equilibrium exists. Furthermore we analyze the superadditivity and stability properties of the game, and show that subadditivity may arise and the recursive core may be empty if the latency functions are not monotone or not continuous.
\end{abstract}

Keywords Cooperative game theory - Partition function form games . Routing · Externalities

Dávid Csercsik at Pázmány Péter Catholic University, Faculty of Information Technology, P.O. Box 278, H-1444 Budapest

Tel.: +36-1 8864700

Fax: +36-1 8864724

and at Game Theory Research Group, Centre for Economics and Regional Science, Hungarian Academy of Sciences Budaörsi 45., H-1112 Budapest.

Tel.: +36-1 3092652

Fax: +36-1 3092650

E-mail: csercsik@scl.sztaki.hu

- Balázs Sziklai at Game Theory Research Group, Centre for Economics and Regional Science, Hungarian Academy of Sciences Budaörsi 45., H-1112 Budapest.

E-mail: sziklai.balazs@krtk.mta.hu 


\section{Introduction}

Problems concerning congested networks originate traditionally from the field of engineering (Altman et al, 2006). Probably this is the reason why noncooperative approach is more wide-spread among the researchers of the subject $^{1}$. The first general model is due to Wardrop (1952), where the network is represented by a graph, while routing tasks are assigned to a subset of nodes and latency functions are defined on the edges. Furthermore it is assumed that the traffic can be divided into infinitesimally small parts which therefore can be represented as flows in the graph. The objective of these infinitesimally small pieces or individuals is to minimize the experienced latency.

Many equilibrium notions were introduced to analyze such congested networks (for a comprehensive study see Roughgarden (2005, 2006)). Most of the literature focuses on Nash equilibria and a related concept the so-called Price of Anarchy (Feldmann et al, 2003). In a congested network Nash equilibrium (NE) is reached when no individual can obtain a lower latency by unilaterally changing his route. In general, such a NE need not be unique. The Price of Anarchy is the ratio between the social cost of the worst Nash equilibrium and the overall optimum solution. The notion was introduced by Koutsoupias and Papadimitriou (2009) and quickly became popular as it successfully captures the possible suboptimality of NE-points. To resolve such situations Stackelberg routing was introduced (Korilis et al, 1997) in which model a certain ratio of all users are obeying to a central authority whose objective is to drive the traffic toward an equilibrium point with lower social cost. In particular there are two types of players a so-called leader and followers. The goal is to find a strategy for the leader that forces the followers to react in a way that minimizes the total latency in the system. For more on this topic see Karakostas and Kolliopoulos (2009).

A possible logical extension of the above model is to consider more than one leader. In other words there are a few distinguished players that altogether are responsible for the whole traffic in the network. The original Wardrop model is inherently non-cooperative, while a setup where all the players are "leaders" is essentially cooperative. The objective of each player is to route his traffic with minimal cost. Sharing information and cooperation with other agents may result in cost savings, and more efficient utilization of network capacities. Depending on the employed strategy of the agents many possible cooperative games can arise. Our aim is to introduce and analyze these wide variety of transferable utility (TU) games. Since the formation of a coalition may affect other players costs via the implied flow and the resulting edge load changes in the network, externalities may arise, thus the underlying games are given in partition function form.

The values of the coalitions are defined as the improvement compared to the reference case, when no cooperation appears. As some coalitions form,

1 Although the cooperative (non-TU) approach is also often used in the case of wireless communication networks Khandani et al (2007) 
routing paths may alter due to the joint optimization process, which may in turn affect the costs of other players via the modified edge latencies. Since such externalities may happen the game is given in partition function form (Thrall and Lucas, 1963).

A scenario where multiple navigation systems are routing their clients on the same traffic network represents a possible application of the defined game theoretic model framework. Novel telecommunication systems can be considered as an other potential application field of the proposed approach (Altman et al, 2006; Khandani et al, 2005, 2007; Devroye et al, 2008)

The structure of the paper is as follows. In section 2 we introduce the notation used, define the partition function form cooperative game on the routing network and summarize the considered routing strategies. The main results are discussed in section 3, where we show that the sequence of iterative predictive strategies of increasing order may converge to routing configuration, which is a Nash equilibrium (NE), but this convergence is not always necessary even if a unique NE exists. Furthermore we analyze the superadditivity and stability properties of the game, and via the recursive core concept we show that overall cooperation may not be always beneficial for the players.

\section{Materials and methods}

In this section we define delivery games and introduce the traffic routing and game theoretic framework that is needed to analyze such games. We made an effort to keep the notational traditions of both fields. To make it more legible we employ the standard that the upper index always refers to some player or a coalition and in case of flows the lower index is always some edge or a path.

First let us recall some basic notions of cooperative games. A cooperative game with transferable utility or simply a $T U$-game is an ordered pair $(N, v)$ consisting of the player set $N=\{1,2, \ldots, n\}$ and a characteristic function $v: 2^{N} \rightarrow \mathbb{R}$ with $v(\emptyset)=0$. The value $v(S)$ is regarded as the worth of coalition $S$. The members of $S$ can achieve this value by cooperating regardless of how players outside the coalition react. In a partition function form (PFF) game $v(S)$ depends also on the partition to where $S$ belongs (Thrall and Lucas, 1963). Let $\Pi(N)$ denote the set of partitions of $N$. An embedded coalition is a pair $(S, \pi)$, where $S \in 2^{N} \backslash\{\emptyset\}$, and $\pi \in \Pi(N)$ is such that $S \in \pi$. We denote by $\mathfrak{C}(N)$ the set of embedded coalitions on $N$. Then a partition function form game is a pair $(N, V)$ where $V: \mathfrak{C}(N) \rightarrow \mathbb{R}$. For a more thorough ${ }^{2}$ definition see (Grabisch and Funaki, 2012).

2 The literature often uses a simplified formalization, namely $V: \pi \rightarrow\left(2^{N} \rightarrow \mathbb{R}\right)$ where the partition function assigns a characteristic functions $\left(v_{\pi}\right)$ to each partition $\pi \in \Pi(N)$. This notation is more intuitive, but misleading a little bit as $v_{\pi}$ is not a proper characteristic function, since $v_{\pi}(T)$ is not defined for $T \notin \pi$. For sake of convenience as well as clarity throughout the paper we will regard $V$ as a mapping from partitions to characteristic functions. 
For $S \in \pi$, the worth of $V(S, \pi)$ denotes the amount that the players in $S$ can guarantee themselves by cooperating, when the coalition $S$ is embedded in the partition $\pi$.

According to the transferable utility assumption, cooperating players may redistribute the wealth gained by cooperation among themselves. The redistribution is formalized via the concept of payoffs. We call the pair $\omega=(x, \pi)$ an outcome, where $\pi \in \Pi(N)$ is a partition and $x=\left(x^{1}, \ldots, x^{n}\right) \in \mathbb{R}^{N}$ is a payoff vector satisfying feasibility; $\sum_{i \in S} x^{i}=V(S, \pi)$ for all $S \in \pi$. Let us denote the the set of outcomes in $(N, V)$ by $\Omega(N, V)$. Next we define the concepts, which will be required for the definition of the PFF delivery game.

\subsection{The Network and routing}

The game takes place on a network $\Gamma$, which is a pair $(G, l)$, represented by a directed graph $G(W, E)$, described by the set of vertices and edges respectively, and a set of edge latency functions $l=\left\{l_{e} \mid e \in E\right\}$. The deliveries of the players are represented as flows in $G$. Latency functions describe how the latency of a certain edge depends on its actual resulting flow. It is commonly accepted to make some constrains on the latency function, such as non-negativity, differentiability and non-decreasingness. We will only assume non-negativity thus $l_{e}: \mathbb{R}_{\geq 0} \rightarrow \mathbb{R}_{\geq 0}$.

A delivery task $\tau=(r, s, t) \in R_{+} \times W \times W$ of a player is described by a quantity and two nodes (a source and sink respectively). To each player $j \in N$ $k^{j}$ delivery tasks are assigned $\tau^{j}=\cup_{i=1}^{k^{j}}\left\{\left(r_{i}^{j}, s_{i}^{j}, t_{i}^{j}\right)\right\}$. The set of all distinct paths from $s_{i}^{j}$ to $t_{i}^{j}$ is denoted by $\mathcal{P}_{i}^{j}$. Distinct paths do not have to be disjoint in general, in other words, they may have common edges. We denote the flow of player $j$ on path $P$ by $f_{P}^{j}$. Then $\mathcal{P}^{j} \stackrel{\text { def }}{=} \cup_{i=1}^{k^{j}} \mathcal{P}_{i}^{j}$ and $\mathcal{P} \stackrel{\text { def }}{=} \cup_{j \in N}\left(\mathcal{P}^{j}\right)$. Therefore $\mathcal{P}$ contains all the possible routes between sources and sinks. Players have to distribute their traffic on the available paths for each delivery task. Formally, we say that $f^{j} \stackrel{\text { def }}{=} \cup_{\left(P \in \mathcal{P}^{j}\right)} f_{P}^{j}$ is a feasible solution for player $j$ iff $\sum_{P \in \mathcal{P}_{i}^{j}} f_{P}^{j}=r_{i}^{j}$ for all $i \in\left\{1,2, \ldots, k^{j}\right\}$. Regarding cooperating players, the flow of a coalition $S$ is denoted by $f \stackrel{\text { def }}{=} \cup_{j \in S} f^{j}$, and it is feasible if $f^{j}$ is feasible for every $j \in S$. The set of feasible solutions is denoted by $\mathbb{F}$.

\subsection{Routing strategies}

Players and coalitions may route their delivery on the available paths according to different possible strategies. These are shortly described below, and demonstrated in section 3. The expression 'routing strategy' is interpreted in a wide sense, including information and beliefs about other players. The zero order strategy assumes that the players have no information about each other while in other cases the delivery tasks are common knowledge. The strategies presented here are pure in the sense that players may route their deliveries 
distributed among multiple different paths in the same time but they do it with probability 1 . Furthermore we assume that cooperating players are always aware of each other's routing tasks and determine their routing by joint design. We denote a specific routing strategy by $\sigma$ and the set of all possible pure routing strategies by $\Sigma$.

\subsubsection{Evaluation of costs and expected costs}

We will use the following notation. The flow of player $j$ on edge $e$ is composed of the different path flows, which include the edge: $f_{e}^{j}=\sum_{P \in \mathcal{P}^{j}: e \in P} f_{P}$, while the flow of coalition $S$ on edge $e$ is $f_{e}^{S}=\sum_{j \in S} f_{e}^{j}$ (regarding the total flow of edge $e$, for convenience sake instead of $f_{e}^{N}$ we write shortly $f_{e}$ ).

The load of edge $e$ with respect to agent $j$ is the traffic that goes through the edge not counting $f_{e}^{j}$. We denote this by $\lambda_{e}^{j}$, formally $\lambda_{e}^{j}=\sum_{k \neq j} f_{e}^{k}=f_{e}-f_{e}^{j}$. Similarly $\lambda_{e}^{S}=\sum_{T \in \pi, T \neq S} f_{e}^{T}=f_{e}-f_{e}^{S}$. The expected load of edge $e$ with respect to coalition $S$ is the flow that goes through $e$ not counting $f_{e}^{S}$ according to the current knowledge of $S$ (which depends on the coalition structure and $\sigma)$. We denote this by $\widehat{\lambda}_{e}^{S}$.

The expected cost of a coalition $S$ is

$$
c^{\exp }(S)=\sum_{e \in E} l_{e}\left(\widehat{\lambda}_{e}^{S}+f_{e}^{S}\right) \cdot f_{e}^{S} .
$$

Note that the value of (1) depends on the routing strategy the players use and the partition $\pi$ embedding $S$. The resulting cost of the coalition $S$ is

$$
c(S)=\sum_{e \in E} l_{e}\left(f_{e}\right) \cdot f_{e}^{S}
$$

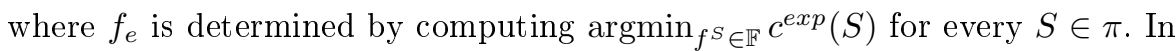
other words each coalition in a given partition has an estimate of how much traffic will appear on certain edges of the network $\left(\widehat{\lambda}_{e}^{S}\right)$ and then determines its routing by minimizing its (expected) cost based on this knowledge. As we will see later the actual cost $c(S)$ induced this way can be quite different than the expected cost. The value of $\widehat{\lambda}_{e}^{S}$ in (1) is determined by the applied strategy as follows.

\subsubsection{Zero order strategy}

This "dummy" strategy assumes that all coalitions neglect the activity of others, and route their deliveries in a way, which is optimal when no other traffic appears on the network. This strategy assumes that non-cooperating players/coalitions have no information of each others routing tasks. In other words $\widehat{\lambda}_{e}^{S}=0$ for each edge $e \in E$ and for each coalition $S \in 2^{N}$. 
2.2.3 First order predictive (FOPS) and n-th order predictive (nOPS) strategy

We define the first order predictive strategy as follows. Every coalition expects the remaining coalitions to route their deliveries according to the zero order strategy, and minimizes his routing costs according to this. This strategy assumes that the coalitions are aware of the other participants delivery contracts. Formally, let us denote the resulting flow of edge $e$ in the zero order routing - when every coalition applies zero order strategy - by $f_{e}\left(\sigma_{0}\right)$. In this case $\widehat{\lambda}_{e}^{S}\left(\sigma_{1}\right)=f_{e}\left(\sigma_{0}\right)-f_{e}^{S}\left(\sigma_{0}\right)$. In the second order predictive strategy (SOPS) all coalitions assume that the remaining ones will route their delivery according to the FOPS etc.

\subsection{Definition of the PFF form delivery game}

Next we define the delivery game, and show how the value of a certain coalition $S$ embedded in a partition $\pi$ can be calculated.

Definition 1 A delivery game $\mathcal{D}=(N, \Gamma, \Delta, \sigma)$ is a 4-tuple consisting of a player set $N$, a network $\Gamma$, a set of delivery tasks $\Delta=\cup_{j} \tau^{j}$ and a routing strategy $\sigma$.

The characteristic functions are defined then as follows. The value of a coalition $S$ in a partition $\pi$ is

$$
v_{(\mathcal{D}, \pi)}(S)=\sum_{j \in S} c_{\left(\mathcal{D}, \pi^{0}\right)}(j)-c_{(\mathcal{D}, \pi)}(S)
$$

where $\pi^{0}$ is the reference, all singleton partition. In other words, the value of a coalition in a certain partition is the difference between the total routing cost of its participants and the overall cost of its members in the all singleton partition. We will see that the players do not always benefit by forming a coalition, hence $v$ can be negative.

Finally the partition function related to the delivery game $\mathcal{D}$ is the function $V_{\mathcal{D}}(\pi)$ that assigns to each partition $\pi \in \Pi(N)$ the characteristic function $v_{(\mathcal{D}, \pi)}(S)$. To simplify the notation, we omit the lower index $(\mathcal{D}, \pi)$ in the case of the cost, expected cost and characteristic functions from now on.

\subsubsection{Routing under Nash equilibrium}

Having defined the game, we can expand our list of possible strategies with another one. Let $A$ be an algorithm that computes a NE for a given routing problem $(N, \Gamma, \Delta)$. Furthermore let $\sigma(A)$ be the routing strategy that routes the delivery tasks as in the NE computed by $A$. Then $\mathcal{D}(N, \Gamma, \Delta, \sigma(A))$ is a delivery game. The equilibrium strategy of coalition $S$ is denoted by $s_{\sigma(A)}^{S}$. Note that the strategy of $S$ is naturally equivalent to the set of flows of $S$, namely $f^{S}$. 


\section{Results}

In this section we demonstrate the various possibly arising properties of the defined game on various networks and examples.

\subsection{Basic properties of predictive strategies}

The predictive technique is an elemental way to strategically approach a game theoretical problem. The most difficult part is to guess the depth of reasoning of the other players. A fair assumption is that the players think that they go at least one step further than the others. Here we only analyzed the case when the depth of reasoning is the same for all players and coalitions, and every actor thinks that the other players take one step less in the reasoning process. Now we state a straightforward but important result.

Theorem 1 Let $\mathcal{D}$ be a delivery game, $\pi=\left\{S_{1}, S_{2}, \ldots, S_{k}\right\}$ a partition of $N$ and let $s_{\sigma_{n}}=\left(f^{S_{1}}\left(\sigma_{n}\right), f^{S_{2}}\left(\sigma_{n}\right) \ldots, f^{S_{k}}\left(\sigma_{n}\right)\right)$ denote the $n$-th order predictive strategy. If $s_{\sigma_{n+1}}=s_{\sigma_{n}}$ then $s_{\sigma_{m}}=s_{\sigma_{n}}$ for all $m>n$ furthermore the resulting routing will be a Nash equilibrium.

Proof: A routing strategy $\tilde{s}=\left(\tilde{f}^{S_{1}}, \tilde{f}^{S_{2}}, \ldots, \tilde{f}^{S_{k}}\right)$ is a NE if for all $S \in \pi$

$$
\underset{f^{S} \in \mathbb{F}}{\operatorname{argmin}} c_{(\mathcal{D}, \pi)}(S)=\underset{f^{S} \in \mathbb{F}}{\operatorname{argmin}} \sum_{e \in E} l_{e}\left(\tilde{\lambda}_{e}^{S}+f_{e}^{S}\right) \cdot f_{e}^{S}=\tilde{f}^{S} .
$$

where $\tilde{\lambda}_{e}^{S}=\sum_{T \in \pi, T \neq S} \tilde{f}_{e}^{T}$.

If the $\mathrm{n}$-th and the $\mathrm{n}+1$-th order predictive strategies coincide, it means that $f^{S}\left(\sigma_{n}\right)=f^{S}\left(\sigma_{n+1}\right)$ for all $S \in \pi$, thus the expected and actual load of any edge $e$ is the same. Formally

$$
\widehat{\lambda}_{e}^{S}\left(\sigma_{n+1}\right)=\sum_{T \in \pi, T \neq S} f_{e}^{T}\left(\sigma_{n}\right)=\sum_{T \in \pi, T \neq S} f_{e}^{T}\left(\sigma_{n+1}\right)=\widehat{\lambda}_{e}^{S}\left(\sigma_{n+2}\right) .
$$

It follows that $s_{\sigma_{m}}=s_{\sigma_{n}}$ for all $m>n$. By the definition of the expected cost

$$
\begin{aligned}
f^{S}\left(\sigma_{n+1}\right)=\underset{f^{S} \in \mathbb{F}}{\operatorname{argmin}} c_{\sigma_{n+1}}^{e x p}(S) & =\underset{f^{S} \in \mathbb{F}}{\operatorname{argmin}} \sum_{e \in E} l_{e}\left(\widehat{\lambda}_{e}^{S}\left(\sigma_{n+1}\right)+f_{e}^{S}\right) f_{e}^{S}= \\
& =\underset{f^{S} \in \mathbb{F}}{\operatorname{argmin}} \sum_{e \in E} l_{e}\left(\sum_{T \in \pi, T \neq S} f_{e}^{T}\left(\sigma_{n}\right)+f_{e}^{S}\right) \cdot f_{e}^{S}= \\
& =\underset{f^{S} \in \mathbb{F}}{\operatorname{argmin}} \sum_{e \in E} l_{e}\left(\sum_{T \in \pi, T \neq S} f_{e}^{T}\left(\sigma_{n+1}\right)+f_{e}^{S}\right) \cdot f_{e}^{S} .
\end{aligned}
$$

for all $S \in \pi$, hence it is indeed a NE.

We can obtain a useful corollary of Theorem 1 by reinterpreting the players strategy. We can think of $f^{S}$ as a $|\mathcal{P}|$ dimensional vector. The coordinates of 
$f^{S}$ corresponds to the flows of the distinct paths between the sources and sinks. In this way it is meaningful to speak about the pointwise convergence of $f^{S}$.

Corollary 1 Let $\mathcal{D}$ be a delivery game with continuous latency functions. If $\lim _{n \rightarrow \infty} s_{\sigma_{n}}=\left(\tilde{f}^{S_{1}}, \tilde{f}^{S_{2}}, \ldots, \tilde{f}^{S_{k}}\right)=\tilde{s}$ where $\tilde{f}^{S_{i}} \in \mathbb{R}^{\mathcal{P}}$ for $i=1,2, \ldots, k$ then $\tilde{s}$ is a NE.

In other words if the flows of increasing order predictive strategies are convergent, they converge to a NE point.

\subsection{Externalities and the convergence of nOPS to NE}

Let us consider network 1 depicted in Fig. 1. We take into account 3 players in order to be able to demonstrate the appearing externalities in the game. In this simple example all players have one delivery task, and the nodes corresponding to the sinks and sources are disjoint.

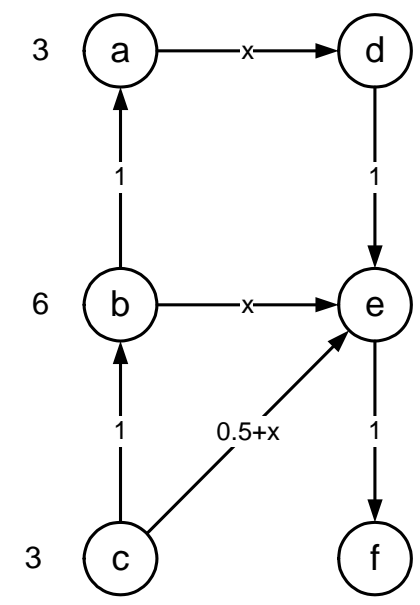

(3)

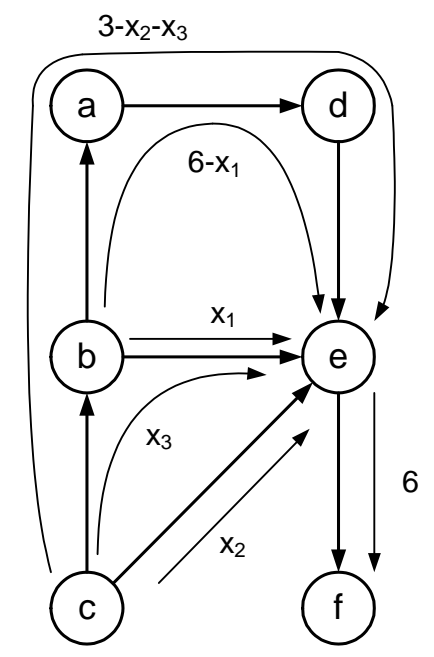

Fig. 1 The basic structure of the network 1, and the possible routing alternatives of the players. The numbers with and without parentheses quantify sinks and sources respectively. Player 2 can route his delivery of 6 units via two ways (the distribution among the two optional paths is described by $x_{1}$ ), while player 3 can route his delivery of 6 units via three ways (the distribution among the three optional paths is described by $x_{2}$ and $x_{3}$ )

Let us suppose the following delivery tasks: $\tau^{1}=(3, a, d), \tau^{2}=(6, b, f)$, $\tau^{3}=(3, c, e)$. Player one has no choice $\left(\left|\mathcal{P}^{1}\right|=1\right)$, player 2 has two possible options $\left(\left|\mathcal{P}^{2}\right|=2\right)$, and thus has one decision variable $x_{1}$, which describes the proportion regarding the distribution of his delivery among the two available 
paths. Player 3 has 3 available paths $\left(\left|\mathcal{P}^{3}\right|=3\right)$, thus he has two decision variables $\left(x_{2}\right.$ and $\left.x_{3}\right)$.

The detailed calculations of this example can be found in Appendix A. The resulting partition function in the case of zero order strategy is summarized in Table 1.

\begin{tabular}{|c|c|}
\hline partition $(\pi)$ & values of coalitions $(v(S))$ \\
\hline$\{1\},\{2\},\{3\}$ & $0,0,0$ \\
$\{1,2\},\{3\}$ & $2.625,-0.75$ \\
$\{1,3\},\{2\}$ & $1.8437,0.1875$ \\
$\{1\},\{2,3\}$ & $0.75,7.125$ \\
$\{1,2,3\}$ & 12.375 \\
\hline
\end{tabular}

Table 1 The resulting partition function of network 1 in the case zero order strategy.

Table 1 clearly demonstrates the emergence of both positive and negative externalities in the case of zero order strategy. As coalitions $\{1\}$ and $\{2\}$ merge, it implies a negative externality on player 3 , while in contrast the merging of coalitions $\{1\}$ and $\{3\}$ or $\{2\}$ and $\{3\}$ is beneficial for the player not included in the cooperation (player 2 and 1 respectively).

\subsubsection{The convergence of nOPS to Nash equilibrium}

After the calculation of the FOPS, we are able to analyze the higher order strategies in the case of various coalition structures. Tables 2 and 3 summarize, how the resulting routing variables, and cost of the coalitions change, while consecutively applying higher order strategies.

\begin{tabular}{|c|c|c|c|c|c|c|c|c|c|}
\hline Partition & $\multicolumn{3}{|c|}{1},\{2\},\{3\}$ & $\multicolumn{3}{|c|}{1,2},\{3\}$ & $\multicolumn{3}{|c|}{1,3},\{2\}$ \\
\hline Strategy & $x_{1}$ & $x_{2}$ & $x_{3}$ & $x_{1}$ & $x_{2}$ & $x_{3}$ & $x_{1}$ & $x_{2}$ & $x_{3}$ \\
\hline Zero order & 3.5 & 1.5 & 1.25 & 4.25 & 1.5 & 1.25 & 4 & 1.63 & 1.38 \\
\hline 1 (FOPS) & 4 & 2.5 & 0.5 & 4.75 & 2.5 & 0.5 & 4 & 2.5 & 0.5 \\
\hline 2 & 4.13 & 2.63 & 0.38 & 4.86 & 2.81 & 0.19 & 4.13 & 2.63 & 0.38 \\
\hline 3 & 4.16 & 2.66 & 0.34 & 4.95 & 2.84 & 0.16 & 4.16 & 2.66 & 0.34 \\
\hline 4 & 4.16 & 2.66 & 0.34 & 4.96 & 2.86 & 0.14 & 4.16 & 2.66 & 0.34 \\
\hline 5 & 4.17 & 2.67 & 0.33 & 4.97 & 2.87 & 0.13 & 4.17 & 2.67 & 0.33 \\
\hline 6 & 4.17 & 2.67 & 0.33 & 4.97 & 2.87 & 0.13 & 4.17 & 2.67 & 0.33 \\
\hline 7 & 4.17 & 2.67 & 0.33 & 4.97 & 2.87 & 0.13 & 4.17 & 2.67 & 0.33 \\
\hline 8 & 4.17 & 2.67 & 0.33 & 4.97 & 2.87 & 0.13 & 4.17 & 2.67 & 0.33 \\
\hline
\end{tabular}

Table 2 The evolution of routing variables $\left[x_{1}, x_{2}, x_{3}\right]$ of network 1 towards NEs as the order of strategies increased.

Let us note that the resulting NE coincides in the case of the all-singleton partiton and $\{1,3\}\{2\}$.

As we can see in tables 2 and 3 , all coalition structures reach the Nash equilibrium with the accuracy of $\varepsilon=10^{-2}$ in the 5 th iteration. 


\begin{tabular}{|c|c|c|c|c|c|c|c|}
\hline Partition & $\multicolumn{3}{|c|}{1},\{2\},\{3\}$ & $\multicolumn{2}{|c|}{1,2},\{3\}$ & $\multicolumn{2}{|c|}{1,3},\{2\}$ \\
\hline Strategy & $c_{1}$ & $c_{2}$ & $c_{3}$ & $c_{\{1,2\}}$ & $c_{3}$ & $c_{\{1,3\}}$ & $c_{2}$ \\
\hline Zero order & 17.25 & 42 & 12.62 & 56.63 & 13.38 & 28.03 & 41.81 \\
\hline 1 (FOPS) & 15 & 38 & 10.25 & 51.5 & 10.63 & 25.25 & 38 \\
\hline 2 & 14.63 & 37.45 & 10.27 & 49.93 & 10.45 & 24.9 & 37.45 \\
\hline 3 & 14.53 & 37.32 & 10.27 & 49.78 & 10.46 & 24.8 & 37.32 \\
\hline 4 & 14.51 & 37.29 & 10.28 & 49.68 & 10.46 & 24.79 & 37.29 \\
\hline 5 & 14.5 & 37.28 & 10.28 & 49.67 & 10.46 & 24.78 & 37.28 \\
\hline 6 & 14.5 & 37.28 & 10.28 & 49.67 & 10.46 & 24.78 & 37.28 \\
\hline 7 & 14.5 & 37.28 & 10.28 & 49.67 & 10.46 & 24.78 & 37.28 \\
\hline 8 & 14.5 & 37.28 & 10.28 & 49.67 & 10.46 & 24.78 & 37.28 \\
\hline
\end{tabular}

Table 3 The evolution of resulting costs of the coalitions in network 1 towards NEs as the order of strategies increased.

The partition $\{1\},\{2,3\}$ is not of interest, because player 1 has no decision variables, in this case all nOPS with $n>1$ will be the same as the FOPS. The routing in the case of the grand coalition is the same in all cases (consider e.g. $\pi=\{1,2,3\})$ assuming zero order strategy.

As we sill see in section 3.3, the FOPS, SOPS, nOPS sequence of strategies is not necessary convergent. Furthermore, as we will show, a NE may exist in a game with divergent nOPS.

\subsection{Divergent nOPS}

In this section we demonstrate on the widely used Pigou network (Pigou, 1920) that the sequence of the increasing order strategies is not necessary convergent even if a unique NE exists in the game.

\subsubsection{Routing under Nash equilibrium on Pigou's graph}

Let $\Gamma_{P}$ be the well-known example of Pigou i.e. a graph with two parallel edges ( $u$ and $w$ ) connecting two nodes ( $s$ and $t$ ). On the so called upper edge $u$ the latency is constant 1 , on the lower edge $w$ the latency is proportional to the traffic (see Fig. 2). Furthermore let $N=\{1,2, \ldots, n\}$ be the set of players with delivery tasks $\tau^{j}=\left(r^{j}, s, t\right)$ i.e. player $j$ has to route $r^{j}$ amount of traffic from $s$ to $t$. Let $A$ be an algorithm that computes a Nash equilibrium in $\Gamma_{P}$ and let $\sigma(A)$ be the corresponding routing strategy. Therefore $\mathcal{D}\left(\Gamma_{P}, N, \Delta, \sigma(A)\right)$ is a well defined delivery game.

Note, that the latency cost of player $j$ is

$$
c(j)=r^{j}-f_{w}^{j}+f_{w}^{j} \cdot f_{w}=r^{j}+\left(\lambda_{w}^{j}-1\right) \cdot f_{w}^{j}+\left(f_{w}^{j}\right)^{2}
$$

As $r^{j}$ is constant $c(j)$ is uniquely determined by how much the players route on the lower edge. Note that $\sigma(A)=\left(f_{w}^{1}, f_{w}^{2}, \ldots, f_{w}^{n}\right)$ is a NE point if no player $j \in N$ can obtain smaller latency cost by altering his strategy. 


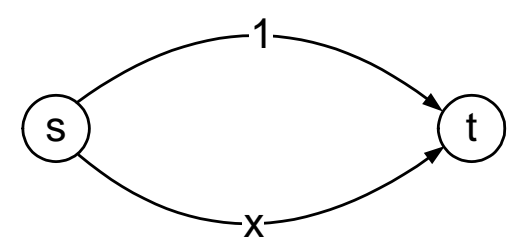

Fig. 2 Network 2: Pigou's graph.

Theorem 2 If $r^{j} \geq \frac{1}{n+1}$ for any player $j \in N$, where $n=|N|$ then there is a unique Nash equilibrium point in $\mathcal{D}\left(\Gamma_{P}, N, \Delta, \sigma(A)\right)$, namely where $f_{w}=\frac{k}{k+1}$ and $f_{w}^{S}=\frac{1}{k+1}$ for any $S \in \pi$, where $k=|\pi|$.

Proof: It is enough to prove for the singleton partition. For other partitions the theorem follows from the fact that each coalition can be considered as a separate player and if $r^{j} \geq \frac{1}{n+1}$ for all $j \in N$ then $r^{S}=\sum_{j \in S} r^{j} \geq \frac{1}{n+1}$ for all $S \in \pi$.

Suppose players follow the same strategy. Then each player $j \in N$ routes $r^{j}-x$ amount of traffic on the upper and $x$ amount on the lower edge. This is a Nash equilibrium point if for any real number $\delta \in \mathbb{R}$, such that $0 \leq x+\delta \leq r^{j}$, if player $j$ routes $\delta$ amount of traffic in a different way, his individual cost is increasing. Formally

$$
r^{j}-(x+\delta)+(x+\delta)(n \cdot x+\delta) \geq r^{j}-x+(x)(n \cdot x) .
$$

We can rewrite the above condition as follows.

$$
\delta^{2}+\delta \cdot x \cdot(n+1)-\delta \geq 0
$$

Which yields

$$
\frac{1-|\delta|}{n+1} \leq x \leq \frac{1+|\delta|}{n+1}
$$

for any real number $\delta$. We can conclude that $x=\frac{1}{n+1}$ is a Nash equlibrium strategy for any number of player $n$.

Now we prove that this is a unique NE point. First suppose that $f_{w}<\frac{n}{n+1}$. In particular let $f_{w}=\frac{n}{n+1}-\epsilon_{1}$. Then there exists $j$ such that $f_{w}^{j}=\frac{1}{n+1}-\epsilon_{2}$ where $\epsilon_{2}$ along with $\epsilon_{1}$ are some positive real numbers. Let $m \stackrel{\text { def }}{=} \min \left(\epsilon_{1}, \epsilon_{2}\right)$. Now increasing $f_{w}^{j}$ by $m$ decreases $c(j)$.

$$
r^{j}-\left(f_{w}^{j}+m\right)+\left(f_{w}^{j}+m\right) \cdot\left(f_{w}+m\right) \leq r^{j}-f_{w}^{j}+f_{w}^{j} \cdot f_{w}
$$

Which is equivalent to

$$
\left(f_{w}^{j}+m\right) \cdot\left(f_{w}+m-1\right) \leq f_{w}^{j} \cdot\left(f_{w}-1\right)
$$


For instance if $m=\epsilon_{2}$

$$
\begin{gathered}
\left(\frac{1}{n+1}-\epsilon_{2}+m\right) \cdot\left(\frac{n}{n+1}-\epsilon_{1}+m-1\right) \leq\left(\frac{1}{n+1}-\epsilon_{2}\right) \cdot\left(\frac{n}{n+1}-\epsilon_{1}-1\right) \\
\left(\frac{1}{n+1}\right) \cdot\left(\frac{n}{n+1}-\epsilon_{1}+\epsilon_{2}-1\right) \leq\left(\frac{1}{n+1}-\epsilon_{2}\right) \cdot\left(\frac{n}{n+1}-\epsilon_{1}-1\right) \\
\frac{\epsilon_{2}}{n+1} \leq \frac{-n \cdot \epsilon_{2}}{n+1}+\epsilon_{1} \cdot \epsilon_{2}+\epsilon_{2} \\
0 \leq \epsilon_{1} \cdot \epsilon_{2}
\end{gathered}
$$

Similar calculations shows that (4) also holds when $m=\epsilon_{1}$. We leave the proof of the case $f_{w}>\frac{n}{n+1}$ to the reader.

\subsubsection{Routing under nOPS strategies on Pigou's graph}

Now we show that for any partition $\pi \in \Pi(N)$ that consists of at least 3 coalition, we can set the delivery tasks in such way that the nOPS strategies do not converge to the NE point in $\mathcal{D}\left(\Gamma_{P}, N, \Delta, \sigma(A)\right)$. It is clear from Theorem 2 that if $\pi$ is fixed then for every $S \in \pi$, the zero order strategy is

$$
s_{0}^{S}= \begin{cases}f_{w}^{S}=\sum_{j \in S} r^{j} & \text { if } \sum_{j \in S} r^{j}<1 / 2 \\ f_{w}^{S}=1 / 2 & \text { otherwise. }\end{cases}
$$

If the number of players and the delivery tasks are such that $\lambda_{w}^{S} \geq 1$ for every $S \in \pi$ then FOPS of every coalition will be to route everything on the upper edge. Then again the SOPS will be the same as the zero order strategy and so on. Therefore nOPS does not necessarily converge as $n$ goes to infinity, even when there is a unique Nash equilibrium point in a given $\mathcal{D}$.

\subsection{Subadditivity}

Intuitively one would expect that the delivery game is superadditive. When a coalition is formed it gains extra information from the new members. The sum $\widehat{\lambda}_{e}^{S}+f_{e}^{S}$ that determines the latency of the edge $e$ seems to be more controllable as $S$ gets larger. However this impression turns out to be wrong. We show two examples of the arising subadditive property for two different strategies.

\subsubsection{An example of subadditivity in the case of zero order strategy}

In this example we demonstrate the subadditivity property on a symmetric three player example assuming zero order strategy. In this three player example the cooperation of any two players implies negative consequences for them and a positive externality for the third player. The explanation for the phenomena 
is that the routing corresponding to the expected minimum cost result in a higher overall cost, implied by the other players' activity.

Let us consider network 3 depicted in Fig. 3 and a delivery game with $\tau^{1}=(2, a, t), \tau^{2}=(2, b, t)$ and $\tau^{3}=(2, c, t)$.

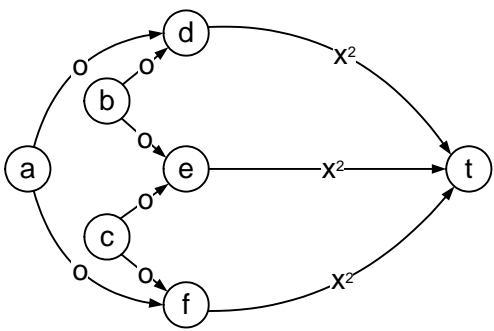

Fig. 3 The basic structure of network 3 .

Coalition structure $\{i\},\{j\},\{k\}$

Because of the symmetry, each player will route his delivery distributed equally between the two available paths. This will result in 2 units of traffic on each line, and a total cost of 8 of each player.

Coalition structure $\{i, j\},\{k\}$

It is easy to see that the cooperating players will route their total delivery distributed equally among the 3 pathways available for them The resulting routing e.g. in the case of the coalition structure $\{1,2\},\{3\}$ will be as depicted in Fig. 4.

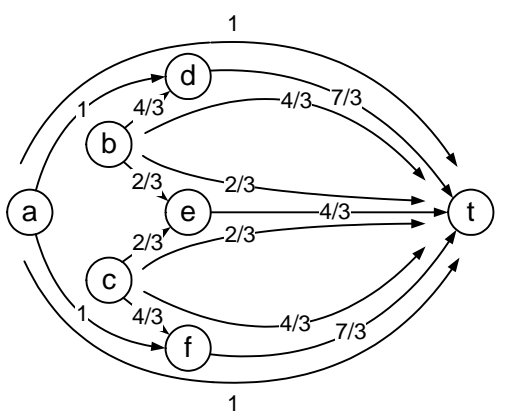

Fig. 4 Routing in the case of coalition structure $\{1,2\},\{3\}$

The cost of the coalition $\{1,2\}$ will be $c(\{1,2\})=16.88$ which is 0.88 units higher than their total cost in singleton configuration $(v(\{i, j\})=-0.88)$. The 
cost of the third player will be $c(3)=2(1+2 / 3)^{2}=5.556$ which implies $v(\{k\})=2.444$

\section{Grand coalition}

In the case of the grand coalition, the resulting routing and routing costs will be as the same as in the case of singleton coalitions, which means that these two coalition configurations are the stable partitions of the game.

\subsubsection{An Example of subadditivity assuming Nash routing strategy}

Let us consider three players with the same delivery task $\tau^{1-3}=(1, s, t)$ on the Pigou network. It follows from Theorem 2 that for coalition structure $\pi^{0}=\{1\}\{2\}\{3\}$ at the NE point every player routes $\frac{1}{4}$ amount of traffic on the lower edge. Therefore each player has $\frac{3}{4} \cdot 1+\frac{1}{4} \cdot \frac{3}{4}=0.9375$ latency cost. For coalition structures $\pi=\{i\}\{j, k\}$ the Nash equilibrium strategies are different as coalition $\{j, k\}$ acts as one player. Therefore at the NE-point there flows $\frac{2}{3}$ traffic on the lower edge. The cost of coalition $\{i\}$ is $\frac{2}{3} \cdot 1+\frac{1}{3} \cdot \frac{2}{3}=0.88$ while $\{j, k\}$ has $\frac{5}{3} \cdot 1+\frac{1}{3} \cdot \frac{2}{3}=1.88^{\circ}$ cost. Since $1.88^{\circ}>2 \cdot 0.9375=1.875$, we can see that the cooperation of players $j$ and $k$ is not beneficial for them, which is an example of subadditivity. If the grand coalition is formed then there goes $\frac{1}{2}$ traffic on the bottom road. The overall cost is $\frac{5}{2} \cdot 1+\frac{1}{2} \cdot \frac{1}{2}=2.75$. Table 4 summarizes the above computation.

\begin{tabular}{|c|c|c|}
\hline partition $(\pi)$ & cost of players $(c(j))$ & values of coalitions $(v(S))$ \\
\hline$\{\mathrm{i}\},\{\mathrm{j}\},\{\mathrm{k}\}$ & $0.9375,0.9375,0.9375$ & $0,0,0$ \\
$\{\mathrm{i}, \mathrm{j}\},\{\mathrm{k}\}$ & $1.8888,0.8888$ & $-0.0138,0.0487$ \\
$\{\mathrm{i}, \mathrm{j}, \mathrm{k}\}$ & 2.75 & 0.0625 \\
\hline
\end{tabular}

Table 4 Routing costs and coalitional values in the case of the Pigou example (network 2) assuming Nash routing.

\subsection{Stability}

To analyze stability and determine a characteristic function for a certain strategy we use the concept of the recursive core Kóczy $(2007,2009)$, that allows the remaining, residual players to freely react and form a core-stable partition before the payoff of the deviating coalition is evaluated.

First we define the residual game over the set $R \subsetneq N$. Let us recall that $\Pi(N)$ denotes the set of partitions of $N$. Assume $\bar{R}=N \backslash R$ have formed $\pi_{\bar{R}} \in \Pi(\bar{R})$. Then the residual game $\left(R, V_{\pi_{\bar{R}}}\right)$ is the PFF game over the player set $R$ with the partition function given by $V_{\pi_{\bar{R}}}\left(S, \pi_{R}\right)=V\left(S, \pi_{R} \cup \pi_{\bar{R}}\right)$. 
Definition 2 (Recursive core Kóczy (2007)) For a single-player game the recursive core is trivially defined. Now assume that the core $R C(N, V)$ has been defined for all games with $|N|<k$ players. For an $|N|$ player game an outcome $(x, \pi)$ is dominated if there exists a coalition $Q$ forming partition $\pi^{\prime}$ and an outcome $\left(y, \pi^{\prime} \cup \pi_{\bar{Q}}\right) \in \Omega(N, V)$, such that $y_{Q}>x_{Q}$ and if $R C\left(\bar{Q}, V_{\pi^{\prime}}\right) \neq \varnothing$ then $\left(y_{\bar{Q}}, \pi_{\bar{Q}}\right) \in R C\left(\bar{Q}, V_{\pi^{\prime}}\right)$. The (recursive) core $R C(N, V)$ of $(N, V)$ is the set of undominated outcomes.

Based on the concept of the Recursive Core, a minimal claim function can be defined, which describes the minimal claim of each coalition in the corresponding PFF game reduced to that coalition. This function, termed $v^{m c}$ in the following, may be applied in the same spirit as a characteristic function, since it assigns a unique value to each coalition, which they can secure for themselves if they deviated. The formal definition of $v^{m c}$ is as follows.

Definition 3 Let us consider the residual game $\left(\bar{S}, V_{\pi_{S}}\right)$ over the player set $\bar{S}$ defined by the partition function $V_{S}\left(R, \pi_{\bar{S}}\right)=V\left(R, \pi_{\bar{S}} \cup S\right)$ where $R \in \pi_{\bar{S}} \in$ $\Pi(\bar{S})$. Let us denote the Recursive Core of the residual game by $R C\left(\bar{S}, V_{S}\right)$. The (pessimistic) minimal claim function $v^{m c}$ can be defined as

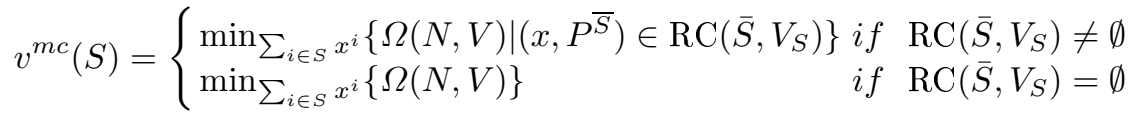

where $v^{m c}(S)$ is the minimal claim of coalition $S$.

With the help of the minimal claim function, a characterization of the Recursive Core can be given as follows.

Lemma 1 The Recursive Core $R C(N, V)$ of the game $(N, V)$ is a collection of Pareto efficient outcomes $(x, \pi) \in \Omega(N, V)$, such that there is no coalition $S$ with $v^{m c}(S)>\sum_{i \in S} x^{i}$.

\subsubsection{The stability of example 1}

According to the concept of the recursive core, the minimal claim functions regarding the strategies of various order can be determined. The minimal claim functions in the case of zero order strategy and FOPS are summarized in Table 5 .

The recursive core can be represented as a polytope in the payoff space. As the order of predictive strategies increase, we may derive the recursive core for each PFF game. Using this method, we get a sequence of recursive corepolytopes, whose geometry may differ in general. We can depict the evolution of the geometry of the recursive core as the order of the applied strategy increases (see Fig. 5). The singleton reference case and so the coalitional values and payoffs are different for each strategy. However as we increase the order of the applied strategy the routing variables and the values of the partition function converge to the $\mathrm{NE}$ and the geometry of the recursive core converge to its final shape. 


\begin{tabular}{|c|c|}
\hline Coalition & Value \\
\hline$\{1\}$ & 0.75 \\
$\{2\}$ & 0.1875 \\
$\{3\}$ & -0.75 \\
$\{1,2\}$ & 2.625 \\
$\{1,3\}$ & 1.8437 \\
$\{2,3\}$ & 7.125 \\
$\{1,2,3\}$ & 12.375 \\
\hline
\end{tabular}$\quad$\begin{tabular}{cc|c|}
\hline Coalition & Value \\
$\{1\}$ & -0.25 \\
$\{2\}$ & 0 \\
$\{3\}$ & -0.375 \\
$\{1,2\}$ & 1.5 \\
$\{1,3\}$ & 0 \\
$\{2,3\}$ & 1.875 \\
$\{1,2,3\}$ & 4 \\
\hline
\end{tabular}

Table 5 Minimal claim functions derived by the recursive core method of example 1 in the case of zero order strategy and FOPS

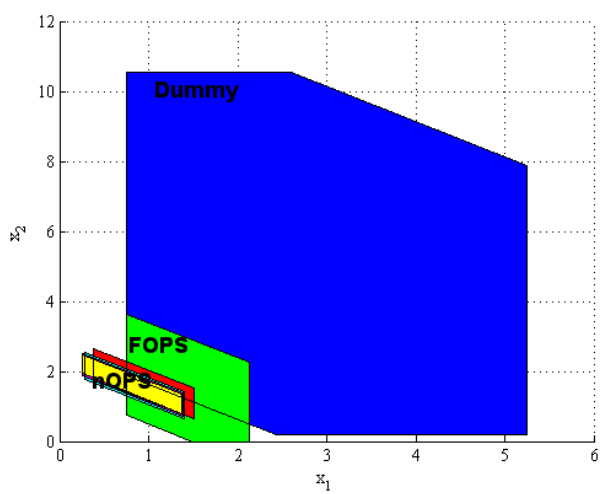

Fig. 5 The projection of the recursive core in the case of various strategies to the plane $x_{3}=0$. The equation $x_{3}=v(\{1,2,3\})-x_{1}-x_{2}$ holds in every case. Let us remember that the cost of the reference case (the all singleton coalitions) according to which the values of the coalitions in different partitions are determined, is different in the case of each strategy - that is the reason why the overall payoff tends to decrease.

\subsubsection{Emptiness of the recursive core}

In this subsection we show two examples, where the recursive core turns out to be empty.

\section{Non-monotone edge latency functions}

In this section we demonstrate that the recursive core may be empty if we assume a network with an edge with non-monotone latency function (depicted in Fig.6), and routing tasks $\delta^{1}=(1, a, t), \delta^{2}=(1, b, t), \delta^{3}=(1, c, t)$.

Since the network is symmetric, we may consider the $\{i\},\{j\},\{k\}$ permutation of players 1,2 and 3 . Zero order strategy and the coalition structure $\{\mathrm{i}\},\{\mathrm{j}\},\{\mathrm{k}\}$ will result in a symmetric configuration, in which each player will route his delivery on the 0.5 latency edge. This results in a total cost of 0.5 of each player.

In contrast, if we assume the coalition structure $\{\mathrm{i}, \mathrm{j}\},\{\mathrm{k}\}$, then $\{\mathrm{i}, \mathrm{j}\}$ will route his traffic on the $(x-2)^{2}$ edge at the cost of $0 \rightsquigarrow v(\{i, j\})=1$, while 

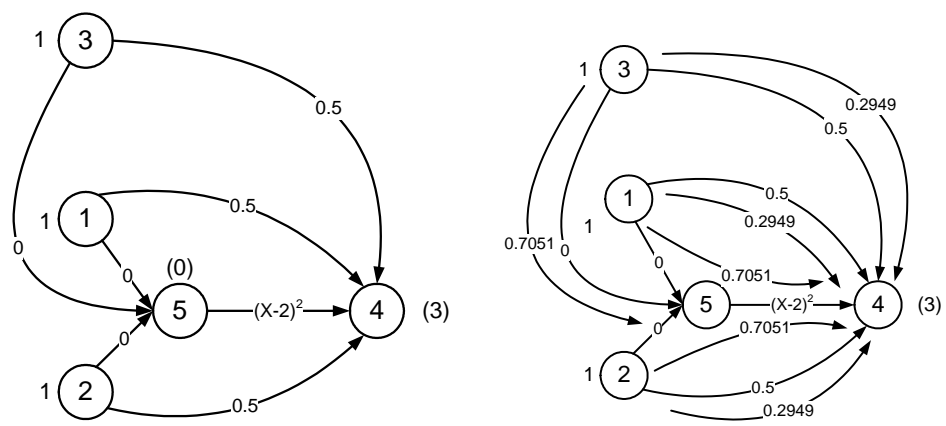

Fig. 6 Example network with non-monotone edge latency function and resulting routing in the case of the grand coalition.

the third player is not affected $(v(k)=0)$. In the case of the grand coalition: $c(\{1,2,3\})=0.4705 \rightsquigarrow v(\{1,2,3\})=1.0295$. It is easy to see that this results in the emptiness of the recursive core.

\section{Non-continuous edge latency functions}

Consider the following example (see Fig. 7$)^{3}$ where $N=\{1,2,3\}$, players follow zero-order strategy and the delivery tasks are $\tau^{1}=(1.4, a, t), \tau^{2}=(1.4, b, t)$ and $\tau^{3}=(1.4, c, t)$.
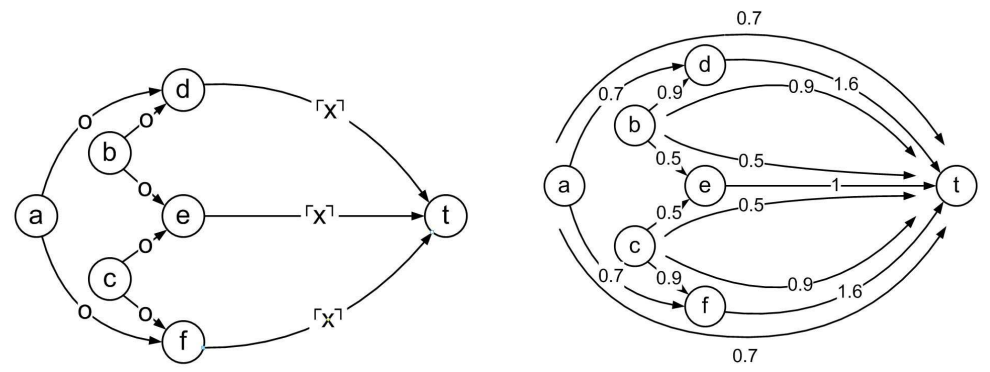

Fig. 7 Example network with non-continuous edge latency function and resulting routing in the case of the grand coalition.

In the case of singleton coalitions each player splits his traffic into two equal parts and sends them on the two possible routes to $t$. In this way each edge with non-zero latency function has a latency cost of 2 (as $\lceil 1.4\rceil=2)$. For partitions $\pi=\{i, j\}\{k\}$, player $i$ and $j$ route on the jointly used edge 1 amount of traffic and send the rest on the other routes. As a result on the other two edge the traffic is increased to 1.6 however this change does not affect the latency cost of these two edges. Finally in the case of grand coalition it is not

$3\lceil x\rceil$ denotes the upper integer part of $x$. 
hard to see that the players can send only 1 amount of traffic with a latency cost of 1 the rest has to be sent for a latency cost of 2 . Therefore the total cost is 7.4. Emptiness of the core follows from the fact that the cost saving of any two person coalition is the same as the cost saving of the grand coalition (see Table 6).

\begin{tabular}{|c|c|c|}
\hline partition $(\pi)$ & cost of players $(c(j))$ & values of coalitions $(v(S))$ \\
\hline$\{\mathrm{i}\},\{\mathrm{j}\},\{\mathrm{k}\}$ & $2.8,2.8,2.8$ & $0,0,0$ \\
$\{\mathrm{i}, \mathrm{j}\},\{\mathrm{k}\}$ & $4.6,2.8$ & 1,0 \\
$\{\mathrm{i}, \mathrm{j}, \mathrm{k}\}$ & 7.4 & 1 \\
\hline
\end{tabular}

Table 6 Emptiness of the core in a network with non-continuous edge latency function.

\section{Conclusions and future work}

In this article a new family of PFF form delivery games on routing networks has been introduced. Various routing strategies have been analyzed, and it has been shown that the sequence of predictive strategies of increasing order may converge to a $\mathrm{NE}$ routing configuration, but it can be also divergent. We have shown on the widely known Pigou network that NE routing may exist in such games, where the nOPS is divergent. We provided examples of subadditive scenarios in various cases, and thus have proven that the defined game is not necessary superadditive. Furthermore we analyzed the stability of the game, and the evolution of the geometry of stable payoff sets via the recursive core concept. In addition we have shown that assuming non monotone or non continuous latency functions the recursive core may be empty.

One straightforward open question is whether the recursive core may be empty if we suppose continuous (strictly) monotone increasing latency functions. An other open problem is how to provide necessary and sufficient conditions for the sequence of iterative strategies to converge to a NE. We hope that the approach of potential methods described in (Nisan et al, 2007) may offer useful tools for the analysis of this problem.

\section{Acknowledgement}

The authors acknowledge the contribution of the members of the Game Theory Research Group, László Á. Kóczy, Helga Habis and Péter Biró. The work has been supported by the Hungarian Academy of Sciences via grant LP-004/2010, by the Hungarian national found OTKA NF 104706, and by TÁMOP-4.2.1/B11/2/KMR-2011-12-0002. The authors dedicate this manuscript to Gábor Holló. 
Appendix A

In this appendix the detailed calculations regarding network 1 depicted in Fig. 1 assuming FOPS strategy can be found, to demonstrate the predictive strategies.

Determination of routing paths according to the zero order strategy

To determine the routing under FOPS strategy, first we have to calculate the resulting routing in the case of zero order strategy. In the case of singleton coalitions, all players neglect the activity of other players, and determine their routing variables $(x)$ by minimizing

$$
c_{(\mathcal{D}, \pi)}^{\exp }(S)=\sum_{e \in E} l_{e}\left(\widehat{\lambda}_{e}^{S}+f_{e}^{S}\right) \cdot f_{e}^{S}
$$

where $\widehat{\lambda_{e}^{S}}=0$ for all $e \in E$ and for all $S \in \pi$. In this case resulting load and latencies of the network will be as depicted in Fig. 8, and listed in Table 2. The routing variables $x$ uniquely determine the edge flows $f_{e}^{S}$.
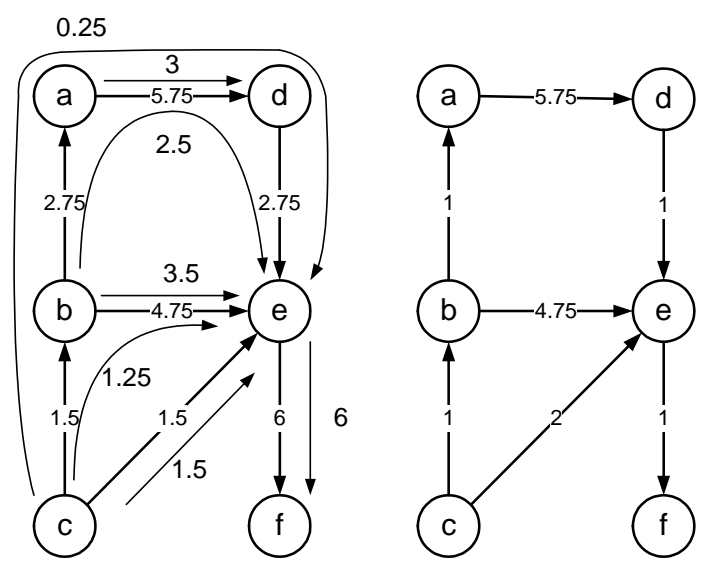

Fig. 8 Resulting routing loads, and edge latencies assuming zero order strategy and singleton coalitions.

The resulting total delivery costs of the coalitions (which are the players themself in this case) can be calculated as:

$$
\begin{aligned}
c(1)= & \left(3+\left(6-x_{1}\right)+\left(3-x_{2}-x_{3}\right)\right) 3 \\
c(2)= & \left(x_{1}+x_{3}\right) x_{1}+2\left(6-x_{1}\right)+\left(6-x_{1}+3-x_{2}-x_{3}+3\right)\left(6-x_{1}\right)+6 \\
c(3)= & \left(0.5+x_{2}\right) x_{2}+\left(3-x_{2}\right)+2\left(3-x_{2}-x_{3}\right)+\left(x_{1}+x_{3}\right) x_{3} \\
& +\left(\left(3-x_{2}-x_{3}\right)+\left(6-x_{1}\right)+3\right)\left(3-x_{2}-x_{3}\right)
\end{aligned}
$$


In the above case the delivery costs will be $c(1)=17.25, c(2)=42, c(3)=$ 12.625, as listed in Table 3. As it can be seen, the zero order strategy (not surprisingly) significantly underestimates the routing costs.

\section{Other coalition structures}

In the case of other partitions, the calculations are similar. Each coalition optimizes the routing variables corresponding to the participating players, taking into account the resulting load the coalition puts on the network. The resulting routing variables and costs are listed in tables 2 and 3.

Determination of routing paths according to the first order predictive strategy (FOPS)

$\pi=\{1\},\{2\},\{3\}$

The route planning of player 1 is still trivial (his expected cost is 8.25 in this case).

Player 2 will assume that player 1 and player 3 will route their delivery according to the zero order strategy. This will result in the minimization of the value of $c(2)$ (see Eq. 5) assuming $\left[\begin{array}{ll}x_{2} & x_{3}\end{array}\right]=\left[\begin{array}{ll}1.5 & 1.25\end{array}\right]\left(c^{\exp }(2)=\right.$ $\left.\left.\left.c(2)\right|_{\left[x_{2}\right.} x_{3}\right]=\left[\begin{array}{ll}1.5 & 1.25\end{array}\right]\right)$ this implies $x_{1}=4$.

According to the zero order routing of players 1 and 2, the expected cost of player 3 will be $c^{\exp }(3)=\left.c(3)\right|_{x_{1}=3.5}$, which is minimal at $\left[\begin{array}{ll}x_{2} & x_{3}\end{array}\right]=\left[\begin{array}{ll}2.5 & 0.5\end{array}\right]$.

In this case the delivery costs will be as follows. $c(1)=15, c(2)=38$, and $c(3)=10.25$. As it can be seen when compared to the zero order strategy, in the case of singleton coalitions the FOPS in this case has reduced the total cost of all players.

$\pi=\{1,2\},\{3\}$

The expected cost of the coalition $\{1,2\}$ is

$$
\left.c^{\exp }(\{1,2\})=c(1)+\left.c(2)\right|_{\left[x_{2}\right.} x_{3}\right]=\left[\begin{array}{ll}
1.5 & 1.25
\end{array}\right]
$$

which is minimal at $x_{1}=4.75$. The routing of player 3 will be as before. The routing costs will be $c(1)=12.75, c(2)=38.75$ and $c(3)=10.625$. Thus the benefit of the cooperation for coalition $\{1,2\}$ is 1.5 , while the value of player 3 is -0.375 in this partition.

$\pi=\{1,3\},\{2\}$

In this case, player 1 and player 3 can not improve their routing, the resulting will be the same as in the singleton case. The expected cost of the coalition $\{1,3\}$ is $c^{\exp }(\{1,3\})=c(1)+\left.c(3)\right|_{x_{1}=3.5}$ which is minimal at $\left[\begin{array}{ll}x_{2} & x_{3}\end{array}\right]=$ $[2.50 .5] . c(1)=15, c(2)=38$, and $c(3)=10.25$.

$\pi=\{1\},\{2,3\}$

The expected cost of the coalition $\{2,3\}$ is $c^{\exp }(\{2,3\})=c(2)+c(3)$ which is minimal at $\left[\begin{array}{lll}x_{1} & x_{2} & x_{3}\end{array}\right]=\left[\begin{array}{lll}4.25 & 3 & 0\end{array}\right] . c(1)=14.25, c(2)=35.875$, and $c(3)=10.5$. This implies a benefit of 1.875 to the coalition $\{2,3\}$. 
$\pi=\{1,2,3\}$

The resulting routing in the case of the grand coalition is the same as under the zero order strategy. This implies here the benefit of 4 for the grand coalition.

\section{References}

Altman E, Boulognea T, El-Azouzi R, Jimenez T, LWynter (2006) A survey on networking games in telecommunications. Computers \& Operations Research 33:286 - 311

Devroye N, Vu M, Tarokh V (2008) Cognitive radio networks: Highlights of information theoretic limits, models and design. IEEE Signal Processing Magazine 25:12-23

Feldmann R, Gairing M, Lucking T, Monien B, Rode M (2003) Selfish routing in non-cooperative networks: A survey. In: Rovan B, Vojtás P (eds) Mathematical Foundations of Computer Science 2003, Lecture Notes in Computer Science, vol 2747, Springer Berlin / Heidelberg, pp 21-45

Grabisch M, Funaki Y (2012) A coalition formation value for games in partition function form. European Journal of Operational Research 221(1):175-185

Karakostas G, Kolliopoulos S (2009) Stackelberg strategies for selfish routing in general multicommodity networks. Algorithmica 53:132 - 153, DOI $10.1007 / \mathrm{s} 00453-007-9018-5$

Khandani A, Modiano E, Abounadi J, Zheng L (2005) Cooperative routing in wireless networks. In: Szymanski B, Bulent Y (eds) Advances in Pervasive Computing and Networking, Springer US, pp 97-117

Khandani A, JAbounadi, EModiano, LZheng (2007) Cooperative routing in static wireless networks. IEEE Transactions on Communications 55:21852192

Kóczy LÁ (2007) A recursive core for partition function form games. Theory and Decision 63(1):41-51, DOI 10.1007/s11238-007-9030-x

Kóczy LÁ (2009) Sequential coalition formation and the core in the presence of externalities. Games and Economic Behavior 66(1):559-565

Korilis Y, Lazar A, Orda A (1997) Achieving network optima using stackelberg routing strategies. IEEE/ACM Transactions on Networking 5:161 -173

Koutsoupias E, Papadimitriou C (2009) Worst-case equilibria. Computer Science Review 3(2):65 - 69, DOI 10.1016/j.cosrev.2009.04.003, URL http://www.sciencedirect.com/science/article/pii/S1574013709000203

Nisan N, Roughgarden T, Tardos E, Vazirani V (2007) Algorithmic Game Theory. Cambridge University Press, 32 Avenue of the Americas, New York, NY 10013-2473, USA

Pigou A (1920) The Economics of Welfare. Macmillan, London

Roughgarden T (2005) Selfish Routing and the Price of Anarchy. MIT Press, 55 Hayward Street Cambridge, MA 02142-1493 USA

Roughgarden $T$ (2006) Selfish routing and the price of anarchy. Tech. rep., Department of Computer Science, Stanford University, URL http://theory.stanford.edu/ tim/papers/optima.pdf 
Thrall RM, Lucas WF (1963) n-person games in partition function form. Naval Research Logistics Quarterly 10(4):281-298

Wardrop J (1952) Some theoretical aspects of road traffic research communication networks. Proc Inst Civ Eng 1:325 - 378 